\title{
Transmit Antenna Selection Schemes for NOMA with Randomly Moving Interferers in Interference-Limited Environment
}

\author{
Dinh-Thuan Do ${ }^{1, * \mathbb{C}}$, Thanh-Luan Nguyen ${ }^{2}$ and Byung Moo Lee ${ }^{3, *(\mathbb{C})}$ \\ 1 Wireless Communications Research Group, Faculty of Electrical and Electronics Engineering, \\ Ton Duc Thang University, Ho Chi Minh City 700000, Vietnam \\ 2 Faculty of Electronics Technology, Industrial University of Ho Chi Minh City (IUH), \\ Ho Chi Minh City 700000, Vietnam; nguyenthanhluan@iuh.edu.vn \\ 3 School of Intelligent Mechatronics Engineering, Sejong University, Seoul 05006, Korea \\ * Correspondence: dodinhthuan@tdtu.edu.vn (D.-T.D.); blee@sejong.ac.kr (B.M.L.)
}

Received: 30 November 2019; Accepted: 24 December 2019; Published: 27 December 2019

check for updates

\begin{abstract}
In this paper, non-orthogonal multiple access (NOMA) is studied at downlink under impact of surrounding interference. This study benefits the practical NOMA system since spatially random interference is adopted. More specifically, we consider the antenna selection strategy applied at the base station and compare the performance of two users. By applying a stochastic geometry-based model, homogeneous Poisson point process (PPP) is employed to consider the spatial topology of interference which is located near to users, and such a model is extremely suitable for practical consideration. We first consider outage probability and then ergodic capacity is examined as main metrics to recommend such model in practice. According to the considered antenna section scheme of the base station, we compare these schemes related to selected antenna serving each user. To confirm exactness of derived expressions, we perform Monte Carlo simulations to verify the analytical results.
\end{abstract}

Keywords: transmit antenna selection; non-orthogonal multiple access; homogeneous Poisson point process; outage probability; ergodic capacity

\section{Introduction}

Wireless communication technology needs higher wireless spectrum efficiency in emerging schemes to implement in applications. As one of the most precious resources, a higher spectrum can be achieved in non-orthogonal multiple access (NOMA) and is suitable to improve the use of wireless spectrum in $5 \mathrm{G}$ networks. In NOMA, to transmit signal from the base station (BS) to multiple destinations, one needs a single frequency spectrum. Hence, to overcome spectrum limitation existing in the traditional OMA systems, the NOMA system provides an improvement of spectral efficiency. Different power factors allocated to users, in which the user experiences a bad channel since it locates farther from the BS while the user that is located near the BS benefits from better channel conditions. Therefore, NOMA exhibits different performance indicators depending on distances between users and the BS. Furthermore, multiple users are multiplexed simultaneously to use the same radio resources by exploiting the power domain [1-5]. One of the advantages from the implement of NOMA from current orthogonal multiple access (OMA) techniques is that massive users are served at the same time. In NOMA systems, successive interference cancellation (SIC) equipping at receivers is required to remove multi-user interference (MUI) caused by the same frequency channel providing multiplexing several users. Many advantages benefit from practical aspects of NOMA systems, where the compatibility of NOMA exhibits system enhancement together with $5 \mathrm{G}$ key technologies such as device-to-device networks [6,7], cognitive radio $[8,9]$, multi-antenna system with beamforming scheme [10-12]. 
System performance of NOMA users are evaluated in several scenarios [13-17]. Having the ability to provide high spectrum efficiency and massive connections, the authors in [13] studied multiple-input multiple-output (MIMO) employing NOMA to fulfill the escalating demands of higher connectivity and data rates. Such emerging wireless applications of MIMO-NOMA are evaluated in derived formulas to indicate improvement in terms of the sum rate and capacity and this model benefits from the antenna with RAS over Rayleigh fading channel. A NOMA downlink system is explored in which a BS is designed with multi-antenna and single-antenna users operate in two multicast groups [14]. They considered a case that one group can afford to be served opportunistically and low-power devices with limited processing capabilities are served in the other group [14]. As a main characteristic, more NOMA users are allowed to access than OMA. Furthermore, the strategy to allocate power to each user and user selection schemes are the main concerns to provide better performance as evaluating NOMA system. In [18], an optimal problem under QoS requirements is considered by minimizing the total transmit power. They introduced how to search for optimal subcarrier assignments for users and suitable allocated power levels are set for subcarriers [18]. Considering downlink and uplink in NOMA network as in [19], two users with the outage probability and the average rate can be achieved to adapt to flexibly meeting various QoS requirements. The exact expressions and approximate expressions at a high signal-to-noise ratio are provided to benefit the proposed system comparing to existing systems [19]. In addition, optimal power allocation within each cell is examined in visible light communication (VLC); multi-cell networks relying on NOMA and such schemes need to perform to obtain a better achievable user rate under user QoS constraints [20]. NOMA is able to be employed with integrated satellite terrestrial networks in [21] in different scenarios of user scheduling schemes. Recently, the authors in $[22,23]$ studied the outage performance of users whose direct links were unavailable in hybrid satellite terrestrial relay networks relying on NOMA. However, the fixed power allocation strategy is implemented in these works [22,23].

Although multiple antenna designs in NOM benefits higher performance gains, unfortunately, a larger number of antennas results in higher cost and complexity in computation. Fortunately, to reduce the undesirable effects of serving multiple antennas at the same time, antenna selection (AS) is introduced as a practical scheme to implement in NOMA. In addition, AS scheme still preserves the diversity and throughput benefits in the scenario of multi-antenna design. For instance, full diversity gain is achieved in AS techniques [24]. In addition, AS in combination with NOMA is introduced in various works [25-28]. In particular, by employing transmit AS (TAS) at the BS, downlink NOMA was investigated in terms of the outage performance [25]. In addition, maximal sum-rate in downlink MIMO-NOMA networks can be obtained in efficient AS; techniques were proposed by recent works [25,27].

More recently, NOMA networks considered some stochastic geometry models to describe the location of nodes and users. The large-scale networks is studied to raise physical layer security of NOMA networks in [29] to enhance the security of a random network and such a system adopts a protected zone around the source node. To evaluate the performance of transmission using a stochastic geometry model, mmWave NOMA networks with random beamforming was investigated in [30]. A downlink transmission with spatially random users is proposed in cooperative NOMA using Decode-and-Forward mode [31]. Both half-duplex and full-duplex for NOMA network are determined under a model of stochastic geometry and in which the authors further evaluate the performance of a pair of relay selection schemes [32]. Although most papers in the literature have explored the NOMA networks, lack of evaluation in case of random locations of users. For example, the results in [13-15] only consider the static models wherein the static locations of users are assumed. To the best of the authors' knowledge, few works examine the situation of random interference surrounding users. More specifically, the location of interference is random in practical and related investigations are very limited. We note that the authors in [32] did not evaluate the situation of degradation in the performance of two user pairing strategies under the impact of external interference. They provided two user pairing modes, namely random and distance-based user pairing. For a specific user pairing strategy, we employ tools from stochastic geometry to derive the outage probability of the downlink 
NOMA scheme. However, open problems as the interference surrounding users are generally operated in any location. In the literature, various works have been conducted to capture the randomness of the surrounding interferers via Poisson point process (PPP) [33-36]. Moreover, Lai X. et al. [36] studied the cooperative relaying networks in which the interference influence the performance at each relay. The interference model in [36] subjected to spatial separation constraints, in which each relay needs to be distant from one another otherwise inadvertently leads to high performance loss [37]. Due to the many applications of NOMA, the paired users are usually separated in terms of distance, e.g., a cell-center and a cell-edge user, thus making it feasible to implement the Poisson interference models into consideration.

Therefore, it is necessary to examine the dynamic location of interference which is located near users in the scenario of downlink NOMA. This study benefits the evaluation of cellular networks on mobile users who met degraded performance. In particular, surrounding interference sources can be examined by statistic methods and then the quality of cellular network is in control by mobile service providers. The main contributions of our work can be summarized as follows:

- We analyze a downlink NOMA network with the degraded performance by randomly distributed interferers located near to users and the number and positions of interferers follow independent PPP. Closed-form expressions of outage probability for the proposed system are given.

- We provide transmit antenna selection schemes, i.e., random antenna selection (RAS) and Best Antenna Selection (BAS), which enable us to improve the quality of transmission and evaluate performance gap on these schemes.

- Lower bound of ergodic capacity and upper bound of outage probability happen at RAS mode for such system model. Moreover, improvement ergodic capacity can be observed via optimal ergodic capacity achieved by heuristic search method. The density of interference make a crucial impact on both outage probability and ergodic capacity performance.

The remainder of this paper is organized as follows. The system model of NOMA as random interference existing near two users is described in Section 2. In Section 3, the Random Antenna Selection (RAS) mode at the base station is introduced via two metrics, i.e., outage probability and ergodic capacity. The best antenna serving each user is presented in Section 4. Numerical and simulation results are discussed in Sections 5 and 6 concludes this paper.

\section{System Model}

This paper proposes a downlink NOMA network consisting a source node equipped with $K$ antenna and two downlink users, namely user $1\left(U_{1}\right)$ and user $2\left(U_{2}\right)$, as in Figure 1 . We assume that each user is affected by a random number of randomly distributed interferer nodes. We assume that the information links, i.e., source-user links, experience independent flat Rayleigh fading. Furthermore, the interference links are assumed to experience independent flat Nakagami- $m$ fading.

In the proposed model, the source node delivers an unit energy superimposed signal $\sqrt{\theta_{1}} x_{1}+\sqrt{\theta_{2}} x_{2}$ through a selected $k$-th antenna to both users, in which $\theta_{n}$ is the power allocation for the $n$-th user, $n \in\{1,2\}$, where $\theta_{1}+\theta_{2}=1$. Hence, the received signal at each $U_{n}$ is given by

$$
y_{n}(k)=\sqrt{P_{S}} \frac{h_{n}^{(k)}}{\sqrt{d_{n}^{\eta}}}\left(\sqrt{\theta_{1}} x_{1}+\sqrt{\theta_{2}} x_{2}\right)+I_{n},
$$

in which $P_{S}$ denotes the transmit power, $h_{n}^{(k)}$ is the channel coefficient between the $k$-th antenna and the $n$-th user, $d_{n}$ is the distance from the source to the $U_{n}, \eta$ is the path-loss exponent and $I_{n}$ specifies the aggregated interference signals at the user $n$. Assuming that the interferers in the vicinity of each user 
are uniformly distributed and follow independent homogeneous Poisson point process $\Phi_{n}$, and $\lambda_{n}$ is the density, i.e., average number of the interferers. Therefore, $I_{n}$ can be formulated as

$$
I_{n}=\sum_{\forall l \in \Phi_{n}} \sqrt{P_{I}} \frac{g_{n}^{(l)}}{\sqrt{r_{n, l}^{\eta}}} x_{n}^{(l)},
$$

in which $P_{I}$ is the transmit power of each interferer, $g_{n}^{(l)}$ and $r_{n, l}$ specify the channel coefficient and the distance from the $l$-th interferer and the $n$-th user, and $x_{n}^{(l)}$ is the transmitted signal from the $l$-th interferer.

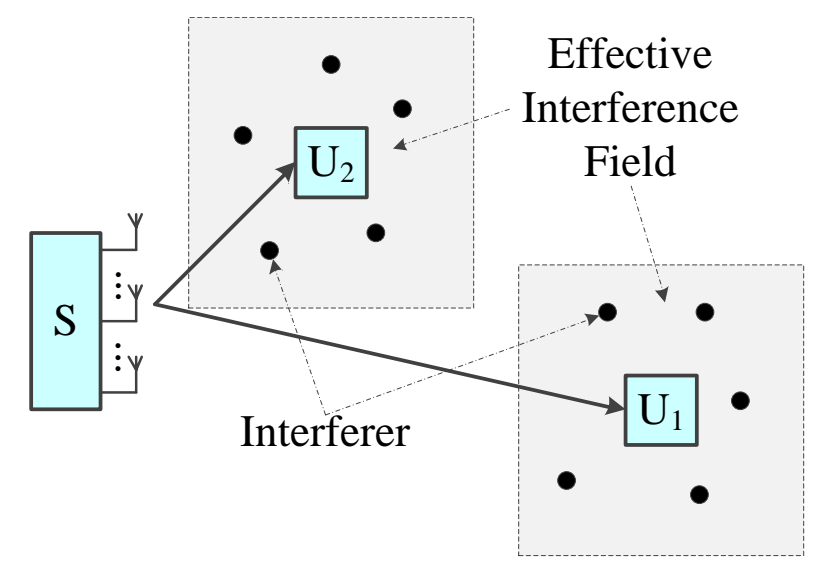

Figure 1. An illustration of the proposed NOMA system in interference-limited environment.

Remark 1. It is noteworthy from [38] that $I_{1}$ and $I_{2}$ might be correlated and causes significant performance loss at the corresponding user. According to [37], such an impact can be mitigated by having the paired users spatially separated. By means of NOMA, $U_{1}$ and $U_{2}$ can be a cell-edge paired with a cell-center user, respectively, which in turns ensures that $U_{1}$ is distant from $U_{2}$. Hence, we can assume that $I_{1}$ and $I_{2}$ are independent.

Furthermore, we assume that

$$
\frac{\left|h_{1}^{(k)}\right|^{2} d_{1}^{-\eta}}{\sum_{\forall l \in \Phi_{1}}\left|g_{1}^{(l)}\right|^{2} r_{1, l}^{-\eta}}<\frac{\left|h_{2}^{(k)}\right|^{2} d_{2}^{-\eta}}{\sum_{\forall l \in \Phi_{2}}\left|g_{2}^{(l)}\right|^{2} r_{2, l}^{-\eta}},
$$

without loss of generality, thus for ensuring fairness, the source node allocates more power for $U_{1}$, i.e., $\theta_{1} \geq \theta_{2}$. The achievable rate at the first user to decode the desired signal while being assisted by the $k$-th antenna is formulated by

$$
R_{1}(k)=\log _{2}\left(1+\frac{\theta_{1} X_{1, k}}{\theta_{2} X_{1, k}+Z_{1}}\right)
$$

where $X_{n, k}=\bar{\gamma}\left|h_{n}^{(k)}\right|^{2} d_{n}^{-\eta}$ with $\bar{\gamma}=P_{S} / P_{I}$ specifies the average signal-to-interference (SIR) ratio, and $Z_{n}=\sum_{\forall l \in \Phi_{n}}\left|g_{n}^{(l)}\right|{ }^{2} r_{n, l}^{-\eta}$. At user $U_{2}$, due to superior allocated power, successive interference cancellation (SIC) is carried out to detect $x_{2}$. First, $U_{2}$ decodes $U_{1}$ 's signal while treating its own signal as an additional interference, thus the instantaneous achievable rate for detecting $x_{1}$ is given as

$$
R_{1 \rightarrow 2}(k)=\log _{2}\left(1+\frac{\theta_{1} X_{2, k}}{\theta_{2} X_{2, k}+Z_{2}}\right) .
$$

After that, $x_{1}$ is then cancelled by means of SIC to decode $x_{2}$ with the following received rate

$$
R_{2}(k)=\log _{2}\left(1+\frac{\theta_{2} X_{2, k}}{Z_{2}}\right)
$$


In the following sections, we introduce three different TAS schemes and the performance associated with each scheme.

\section{Random Antenna Selection (RAS)}

In this strategy, the source randomly selects an antenna to deliver the superimposed message to both users. The performance at each user is introduced as follows.

\subsection{Outage Probability}

At user $U_{1}$, the outage probability is defined via the event this user cannot detect its own signal, i.e., $R_{1}$ falls below a data rate threshold $\bar{R}_{1}$ (bps/Hz). Subsequently, the outage probability at this user while being assisted by the $k$-th antenna is given by

$$
\begin{aligned}
O P_{1}^{R A S}(k) & =\operatorname{Pr}\left(R_{1}<\bar{R}_{1}\right) \\
& =\operatorname{Pr}\left(\frac{X_{1, k}}{Z_{1}}<\frac{\vartheta_{1}}{\theta_{1}-\vartheta_{1} \theta_{2}}\right), \bar{R}_{1}<\frac{\theta_{1}}{\theta_{2}} .
\end{aligned}
$$

in which $\vartheta_{n}=2^{\bar{R}_{n}}-1$. At user $U_{2}$, the outage probability is defined via the events (i) this user cannot decode $x_{1}$ or (ii) this user correctly detects $x_{1}$ but cannot decode $x_{2}$ after successful SIC. Hence, this probability can be formulated as

$$
\begin{aligned}
O P_{2}^{R A S}(k) & =\operatorname{Pr}\left(R_{1 \rightarrow 2}(k)<\bar{R}_{1}\right)+\operatorname{Pr}\left(R_{1 \rightarrow 2}(k)>\bar{R}_{1}, R_{2}(k)<\bar{R}_{2}\right) \\
& =\operatorname{Pr}\left(R_{1 \rightarrow 2}(k)<\bar{R}_{1} \text { or } R_{2}(k)<\bar{R}_{2}\right) \\
& =\operatorname{Pr}\left(\frac{X_{2, k}}{Z_{2}}<\max \left(\frac{\vartheta_{1}}{\theta_{1}-\vartheta_{1} \theta_{2}}, \frac{\theta_{2}}{\theta_{2}}\right)\right), \bar{R}_{1}<\frac{\theta_{1}}{\theta_{2}} .
\end{aligned}
$$

Let $\tau_{1}=\frac{\vartheta_{1}}{\theta_{1}-\vartheta_{1} \theta_{2}}, \tau_{2}=\max \left(\frac{\vartheta_{1}}{\theta_{1}-\vartheta_{1} \theta_{2}}, \frac{\vartheta_{2}}{\theta_{2}}\right)$ and $G_{n}^{(k)}=X_{n, k} / Z_{n}$ be the ordered channel fading gain normalized by the aggregated interference. It can be seen from (8) and (11) that $O P_{n}^{(k)}=F_{G_{n}^{(k)}}\left(\tau_{n}\right)$, where $F_{G_{n}^{(k)}}(\gamma)$ is the $\mathrm{CDF}$ of $G_{n}^{(k)}$. By use of order statistics, $F_{G_{1}^{(k)}}(\gamma)$ and $F_{G_{2}^{(k)}}(\gamma)$ are given by

$$
\begin{gathered}
F_{G_{1}^{(k)}}(\gamma)=\operatorname{Pr}\left(\min \left(\bar{G}_{1}^{(k)}, \bar{G}_{2}^{(k)}\right)<\gamma\right)=1-\left(1-F_{\bar{G}_{1}^{(k)}}(\gamma)\right)\left(1-F_{\bar{G}_{2}^{(k)}}(\gamma)\right), \\
F_{G_{2}^{(k)}}(\gamma)=\operatorname{Pr}\left(\max \left(\bar{G}_{1}^{(k)}, \bar{G}_{2}^{(k)}\right)<\gamma\right)=F_{\bar{G}_{1}^{(k)}}(\gamma) F_{\bar{G}_{2}^{(k)}}(\gamma),
\end{gathered}
$$

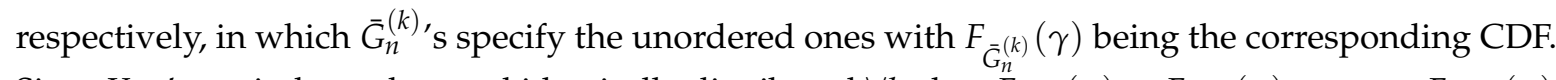
Since $X_{n, k}$ 's are independent and identically distributed $\forall k$, thus $F_{\bar{G}_{n}^{(1)}}(\gamma)=F_{\bar{G}_{n}^{(2)}}(\gamma)=\cdots=F_{\bar{G}_{n}^{(N)}}(\gamma)$ which in turns obtains $F_{G_{n}^{(1)}}(\gamma)=F_{G_{n}^{(2)}}(\gamma)=\cdots=F_{G_{n}^{(N)}}(\gamma)$. For convenience, let $F_{\bar{G}_{n}}(\gamma)=F_{G_{n}^{(k)}}(\gamma)$ and $F_{G_{n}}(\gamma)=F_{G_{n}^{(k)}}(\gamma), \forall k$. The $\mathrm{CDF}$ of the ordered $G^{k}$ is given in the following proposition.

Proposition 1. The CDF of the unordered $\bar{G}_{n}^{(k)}$ is given by

$$
F_{\bar{G}_{n}^{(k)}}(\gamma)=F_{\bar{G}_{n}}(\gamma)=1-\exp (-\underbrace{\frac{\pi \lambda_{n}}{\Gamma\left(\alpha_{n}\right)} \Gamma\left(\alpha_{n}+\frac{2}{\eta}\right) \Gamma\left(1-\frac{2}{\eta}\right)}_{C_{n}}\left(\frac{\gamma}{\mu_{n} \alpha_{n}}\right)^{\frac{2}{\eta}}), \gamma>0 .
$$

in which $\mu_{n}=\bar{\gamma} / d_{n}^{\eta}$ and $\alpha_{n}$ specifies the shape parameter of $\left|g_{n}^{(l)}\right|^{2}, \forall l \in \Phi_{n}$.

Proof: See Appendix A. 
Substituting (11) into (7) and (8), one can obtain the analytical forms for the outage probability at $U_{1}$ and $U_{2}$, respectively. Please note that both $O P_{1}^{R A S}(k)$ and $O P_{2}^{R A S}(k)$ become one $(\forall k)$ when $\bar{R}_{1} \geq \theta_{1} / \theta_{2}$.

\subsection{Ergodic Capacity of $x_{1}$}

Ergodic Capacity of $x_{1}$ while being assisted by the $k$-th antenna is given as

$$
\begin{aligned}
E_{x_{1}}^{R A S}(k) & =\mathbb{E}\left[\log _{2}\left(1+\frac{\theta_{1} X_{1, k}}{\theta_{2} X_{1, k}+Z_{1}}\right)\right] \\
& \stackrel{(a)}{=} \frac{1}{\ln 2} \int_{0}^{\frac{\theta_{1}}{\theta_{2}}} \frac{1}{1+x}\left(1-F_{G_{1}^{(k)}}\left(\frac{x}{\theta_{1}-x \theta_{2}}\right)\right) d x \\
& \stackrel{(b)}{=} \frac{1}{\ln 2} \frac{\theta_{1}}{\theta_{2}-1}\left(\int_{0}^{\infty} \frac{1}{t+\frac{1}{\theta_{2}}} \bar{F}_{G_{1}}(t) d t-\int_{0}^{\infty} \frac{1}{1+t} \bar{F}_{G_{1}}(t) d t\right)
\end{aligned}
$$

in which $(a)$ is obtained by performing integral-by-part while $(b)$ is given via the variable change $t \leftarrow \frac{x}{\theta_{1}-x \theta_{2}}$ and then performs some necessary manipulations.

To derive (14) into closed-form, we consider the following well-known transformations as

$$
\begin{gathered}
e^{-p t^{v}}=G_{0,1}^{1,0}\left(\left.p t^{v}\right|_{0} ^{-}\right), p>0, v>0 \\
\frac{1}{t+1}=G_{1,1}^{1,1}\left(t \mid \begin{array}{l}
0 \\
0
\end{array}\right), \\
\frac{1}{(t+1)^{2}}=G_{1,1}^{1,1}\left(t \mid \begin{array}{c}
-1 \\
0
\end{array}\right), \\
\left(t+\frac{1}{\theta_{2}}\right)^{-1}=\theta_{2} G_{1,1}^{1,1}\left(\begin{array}{l|l}
\theta_{2} t & 0 \\
0
\end{array}\right),
\end{gathered}
$$

in which $G_{p, q}^{m, n}(\cdot \mid \cdot)$ denotes the Meijer's G function, thus the first integral part can be derived as

$$
\begin{aligned}
\int_{0}^{\infty} \frac{1}{1+t} \bar{F}_{G_{1}}(t) d t & =\int_{0}^{\infty} G_{1,1}^{1,1}\left(t \mid \begin{array}{l}
0 \\
0
\end{array}\right) G_{0,1}^{1,0}\left(C_{1}\left(\frac{t}{\mu_{1} \alpha_{1}}\right)^{\frac{2}{\eta}}+\left.C_{2}\left(\frac{t}{\mu_{2} \alpha_{2}}\right)^{\frac{2}{\eta}}\right|_{0} ^{-}\right) d t \\
& =H_{1,2}^{2,1}\left(C_{1}\left(\frac{1}{\mu_{1} \alpha_{1}}\right)^{\frac{2}{\eta}}+C_{2}\left(\frac{1}{\mu_{2} \alpha_{2}}\right)^{\frac{2}{\eta}} \begin{array}{c}
\left(0,2 \eta^{-1}\right) \\
(0,1),\left(0,2 \eta^{-1}\right)
\end{array}\right)
\end{aligned}
$$

in which the last equation is obtained via computation of $H_{p, q}^{m, n}(\cdot \mid \cdot)$ which specifies the Fox H-function. The remaining integrals can be expressed in a closed-form with similar manner. Consequently, the analytical form of the Ergodic Capacity of $x_{1}$ can be given as

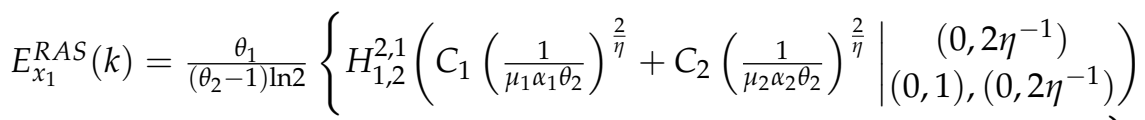

$$
\begin{aligned}
& \left.-H_{1,2}^{2,1}\left(C_{1}\left(\frac{1}{\mu_{1} \alpha_{1}}\right)^{\frac{2}{\eta}}+C_{2}\left(\frac{1}{\mu_{2} \alpha_{2}}\right)^{\frac{2}{\eta}} \mid \begin{array}{c}
\left(0,2 \eta^{-1}\right) \\
(0,1),\left(0,2 \eta^{-1}\right)
\end{array}\right)\right\} \text {. }
\end{aligned}
$$

\subsection{Ergodic Capacity of $x_{2}$}

Ergodic Capacity of $x_{2}$ while being assisted by the $k$-th antenna is given as

$$
E_{x_{2}}^{R A S}(k)=\mathbb{E}\left[\log _{2}\left(1+\frac{\theta_{2} X_{2, k}}{Z_{2}}\right)\right]
$$




$$
\begin{aligned}
& \stackrel{(c)}{=} \frac{1}{\ln 2} \int_{0}^{\infty} \frac{1}{1+x}\left(1-F_{G_{2}^{(k)}}\left(\frac{x}{\theta_{2}}\right)\right) d x \\
& \stackrel{(d)}{=} \frac{1}{\ln 2} \int_{0}^{\infty} \frac{1}{1+x} \bar{F}_{\bar{G}_{1}}\left(\frac{x}{\theta_{2}}\right) d x+\frac{1}{\ln 2} \int_{0}^{\infty} \frac{1}{1+x} \bar{F}_{\bar{G}_{2}}\left(\frac{x}{\theta_{2}}\right) d x \\
& \quad-\frac{1}{\ln 2} \int_{0}^{\infty} \frac{1}{1+x} \bar{F}_{\bar{G}_{1}}\left(\frac{x}{\theta_{2}}\right) \bar{F}_{\bar{G}_{2}}\left(\frac{x}{\theta_{2}}\right) d x .
\end{aligned}
$$

in which $(c)$ is obtained via integration-by-part and $(d)$ is via substituting (10) into (19). Furthermore, by applying (11), we found that the above integrals are similar to (16) and thus can be solved similarly. Therefore, $E_{x_{2}}^{(k)}$ can be derived in closed-form as

$$
\begin{aligned}
E_{x_{2}}^{R A S}(k)=\frac{1}{\ln 2}\{ & H_{1,2}^{2,1}\left(C_{1}\left(\frac{1}{\mu_{1} \alpha_{1} \theta_{2}}\right)^{\frac{2}{\eta}} \mid \begin{array}{c}
\left(0,2 \eta^{-1}\right) \\
(0,1),\left(0,2 \eta^{-1}\right)
\end{array}\right)+H_{1,2}^{2,1}\left(C_{2}\left(\frac{1}{\mu_{2} \alpha_{2} \theta_{2}}\right)^{\frac{2}{\eta}} \mid \begin{array}{c}
\left(0,2 \eta^{-1}\right) \\
(0,1),\left(0,2 \eta^{-1}\right)
\end{array}\right) \\
& \left.-H_{1,2}^{2,1}\left(C_{1}\left(\frac{1}{\mu_{1} \alpha_{1} \theta_{2}}\right)^{\frac{2}{\eta}}+C_{2}\left(\frac{1}{\mu_{2} \alpha_{2} \theta_{2}}\right)^{\frac{2}{\eta}} \mid \begin{array}{c}
\left(0,2 \eta^{-1}\right) \\
(0,1),\left(0,2 \eta^{-1}\right)
\end{array}\right)\right\} .
\end{aligned}
$$

\section{Best Antenna Selection Mode}

\subsection{Best Antenna Serving $U_{1}(B A S-1)$}

In this scheme, the source node selects the best antenna for maximal performance at $U_{1}$. This strategy can optimize the performance of the system in the scenario where $U_{2}$ performance is guaranteed due to superior normalized channel gain and the source prefers a suitable antenna to serve $U_{1}$. Subsequently, the selected antenna, denoted by $k_{1}^{*}$, can be formulated as

$$
k_{1}^{*}=\arg \max _{k=1,2, \ldots, K} G_{1}^{(k)} .
$$

Let $F_{G_{n}}^{\left(k_{n}^{*}\right)}(\gamma)$ be the CDF of $G_{n}^{\left(k_{n}^{*}\right)}$, it immediately follows that $F_{G_{2}}^{\left(k_{1}^{*}\right)}(\gamma)=F_{G_{2}}(\gamma)$, thus the outage probability and the Ergodic Capacity at $U_{2}$ in this scheme is similar to those in $R A S$, i.e., $O P_{2}^{B A S-1}=$ $O P_{1}^{R A S}$ and $E_{x_{2}}^{B A S-1}=E_{x_{2}}^{R A S}$. In addition, the ordered random variables $G_{1}^{\left(k_{1}^{*}\right)}$ can be re-expressed as

$$
G_{1}^{\left(k_{1}^{*}\right)}=\max \left(\min \left(\bar{G}_{1}^{(1)}, \bar{G}_{2}^{(1)}\right), \min \left(\bar{G}_{1}^{(2)}, \bar{G}_{2}^{(2)}\right), \ldots, \min \left(\bar{G}_{1}^{(K)}, \bar{G}_{2}^{(K)}\right)\right) .
$$

It can be noticed that " $\min \left(\bar{G}_{1}^{(k)}, \bar{G}_{2}^{(k)}\right)$ ", where $k \in[1, K]$, are identically distributed and are dependent due to the mutual interference terms, i.e., $I_{1}$ and $I_{2}$. By rules of combinatorics, the CDF of $G_{1}^{\left(k_{1}^{*}\right)}$ can be derived as

$$
\begin{aligned}
F_{G_{1}}^{\left(k_{1}^{*}\right)}(\gamma) & =1+\sum_{k=1}^{K}\left(\begin{array}{l}
K \\
k
\end{array}\right)(-1)^{k} \operatorname{Pr}\left(\bigcap_{t=1}^{k} \min \left(\bar{G}_{1}^{(t)}, \bar{G}_{2}^{(t)}\right)>\gamma\right) \\
& =1+\sum_{k=1}^{K}\left(\begin{array}{l}
K \\
k
\end{array}\right)(-1)^{k} \operatorname{Pr}\left(\min \left(\frac{\min \left(\left(X_{1, t}\right)_{t=1}^{k}\right)}{I_{1}}, \frac{\min \left(\left(X_{2, t}\right)_{t=1}^{k}\right)}{I_{2}}\right)>\gamma\right) \\
& =1+\sum_{k=1}^{K}\left(\begin{array}{l}
K \\
k
\end{array}\right)(-1)^{k} \exp \left(-C_{1}\left(\frac{k \gamma}{\mu_{1} \alpha_{1}}\right)^{\frac{2}{\eta}}-C_{2}\left(\frac{k \gamma}{\mu_{2} \alpha_{2}}\right)^{\frac{2}{\eta}}\right) .
\end{aligned}
$$


Substituting (26) into (13) yields similar integrals as in (16), thus by use of (16), one can obtain the closed-form of the Ergodic Capacity as $U_{1}$ for $B A S-1$ scheme as

$$
\begin{gathered}
E_{x_{1}}^{B A S-1}=-\frac{1}{\ln 2} \frac{\theta_{1}}{\theta_{2}-1} \sum_{k=1}^{K}\left(\begin{array}{l}
K \\
k
\end{array}\right)(-1)^{k}\left\{H_{1,2}^{2,1}\left(C_{1}\left(\frac{k}{\mu_{1} \alpha_{1} \theta_{2}}\right)^{\frac{2}{\eta}}+C_{2}\left(\frac{k}{\mu_{2} \alpha_{2} \theta_{2}}\right)^{\frac{2}{\eta}} \mid \begin{array}{c}
\left(0,2 \eta^{-1}\right) \\
(0,1),\left(0,2 \eta^{-1}\right)
\end{array}\right)\right. \\
\left.-H_{1,2}^{2,1}\left(C_{1}\left(\frac{k}{\mu_{1} \alpha_{1}}\right)^{\frac{2}{\eta}}+C_{2}\left(\frac{k}{\mu_{2} \alpha_{2}}\right)^{\frac{2}{\eta}} \mid \begin{array}{c}
\left(0,2 \eta^{-1}\right) \\
(0,1),\left(0,2 \eta^{-1}\right)
\end{array}\right)\right\} .
\end{gathered}
$$

\subsection{Best Antenna Serving $U_{2}(B A S-2)$}

In this scheme, the source node selects the best antenna for maximal performance at $U_{2}$. This strategy can optimize the performance of the system in the scenario where the performance at $U_{1}$ cannot be guaranteed due to high interference level and/or poor channel condition. Subsequently, the source prefers a suitable antenna to serve $U_{2}$. The selected antenna, denoted by $k_{2}^{*}$, can be formulated as

$$
k_{2}^{*}=\arg \max _{k=1,2, \ldots, K} G_{2}^{(k)} .
$$

It then immediately follows that $F_{G_{1}}^{k_{2}}(\gamma)=F_{G_{1}}(\gamma)$, thus the outage probability and the Ergodic Capacity at $U_{1}$ in this scheme is similar to those in $R A S$, i.e., $O P_{1}^{B A S-2}=O P_{1}^{R A S}$ and $E_{x_{1}}^{B A S-2}=E_{x_{1}}^{R A S}$. In addition, the ordered random variables $G_{2}^{\left(k_{2}^{*}\right)}$ can be re-expressed as

$$
\begin{aligned}
G_{2}^{\left(k_{2}^{*}\right)} & =\max \left(\max \left(\bar{G}_{1}^{(1)}, \bar{G}_{2}^{(1)}\right), \max \left(\bar{G}_{1}^{(2)}, \bar{G}_{2}^{(2)}\right), \ldots, \max \left(\bar{G}_{1}^{(K)}, \bar{G}_{2}^{(K)}\right)\right. \\
& =\max \left(\frac{\max \left(\left(X_{1, k}\right)_{k=1}^{K}\right)}{I_{1}}, \frac{\max \left(\left(X_{2, k}\right)_{k=1}^{K}\right)}{I_{2}}\right)
\end{aligned}
$$

in which the last equality is obtained by using the definition of $\bar{G}_{n}^{(k)}$ and then performing some necessary algebraic transforms. The CDF of $\max \left(\left(X_{1, k}\right)_{k=1}^{K}\right)$, denoted by $F_{M_{n}}(\gamma)$, can be given by

$$
F_{M_{n}}(\gamma)=\left(1-\exp \left(-\frac{\gamma}{\mu_{n}}\right)\right)^{K}, \gamma>0
$$

By use of binomial theorem, one can obtain

$$
F_{M_{n}}(\gamma)=\sum_{k_{n}=0}^{K}\left(\begin{array}{c}
K \\
k_{n}
\end{array}\right)(-1)^{k_{n}} \exp \left(-\frac{k_{n} \gamma}{\mu_{n}}\right), \gamma>0
$$

To perform the remaining analysis, the $\mathrm{CDF}$ of $G_{2}^{\left(k_{2}^{*}\right)}$ need be computed by

$$
F_{G_{2}}^{\left(k_{2}^{*}\right)}(\gamma)=1+\sum_{\substack{k_{1}, k_{2}=0 \\
k_{1}+k_{2} \neq 0}}^{K}\left(\begin{array}{l}
K \\
k_{1}
\end{array}\right)\left(\begin{array}{l}
K \\
k_{2}
\end{array}\right)(-1)^{k_{1}+k_{2}} \exp \left(-C_{1}\left(\frac{k_{1} \gamma}{\mu_{1} \alpha_{1}}\right)^{\frac{2}{\eta}}-C_{2}\left(\frac{k_{2} \gamma}{\mu_{2} \alpha_{2}}\right)^{\frac{2}{\eta}}\right) .
$$

Substituting (32) into (13) yields similar integrals as in (16), thus by use of (16), one can obtain the closed-form of the Ergodic Capacity as $U_{2}$ for $B A S-2$ scheme as

$$
\begin{aligned}
E_{x_{2}}^{B A S-2}= & \frac{1}{\ln 2} \sum_{\substack{k_{1}, k_{2}=0 \\
k_{1}+k_{2} \neq 0}}^{K}\left(\begin{array}{l}
K \\
k_{1}
\end{array}\right)\left(\begin{array}{l}
K \\
k_{2}
\end{array}\right)(-1)^{k_{1}+k_{2}-1} \\
& \times H_{1,2}^{2,1}\left(C_{1}\left(\frac{k_{1}}{\mu_{1} \alpha_{1} \theta_{2}}\right)^{\frac{2}{\eta}}+C_{2}\left(\frac{k_{2}}{\mu_{2} \alpha_{2} \theta_{2}}\right)^{\frac{2}{\eta}} \mid \begin{array}{c}
\left(0,2 \eta^{-1}\right) \\
(0,1),\left(0,2 \eta^{-1}\right)
\end{array}\right) .
\end{aligned}
$$


The optimal sum Ergodic Capacity can be obtained via the following antenna selection strategy

$$
k^{\text {opt. }}=\arg \max _{k=1,2, \ldots, K}\left(\log _{2}\left(1+\frac{\theta_{1} X_{1, k}}{\theta_{2} X_{1, k}+Z_{1}}\right)+\left(\log _{2}\left(1+\frac{\theta_{2} X_{2, k}}{Z_{2}}\right)\right),\right.
$$

in which $k^{\text {opt. }}$ can be effectively achieved by simple search methods, e.g., exhaustive search.

For ease of reference, we provide Table 1 as follows.

Table 1. A summary of the results.

\begin{tabular}{ccccccc}
\hline & \multicolumn{2}{c}{ RAS } & \multicolumn{2}{c}{ BAS-1 (22) } & \multicolumn{2}{c}{ BAS-2 (28) } \\
\hline \multirow{2}{*}{$U_{1}$} & Sim. & Ana. & Sim. & Ana. & Sim. & Ana. \\
\cline { 2 - 7 } & $O P_{1}^{R A S}(1),(7)$ & $F_{G_{1}}\left(\tau_{1}\right),(9)$ & $O P_{1}^{R A S}\left(k_{1}^{*}\right),(7)$ & $F_{G_{1}}^{\left(k_{1}^{*}\right)}\left(\tau_{1}\right),(26)$ & $O P_{1}^{R A S}\left(k_{2}^{*}\right),(7)$ & $F_{G_{1}}\left(\tau_{1}\right),(9)$ \\
& $E_{x_{1}}^{R A S}(1),(12)$ & $E_{x_{1}}^{R A S}(1),(17)$ & $E_{x_{1}}^{R A S}\left(k_{1}^{*}\right),(12)$ & $E_{x_{1}}^{B A S-1},(27)$ & $E_{x_{1}}^{R A S}\left(k_{2}^{*}\right),(12)$ & $E_{x_{1}}^{R A S}(1),(17)$ \\
\hline \multirow{2}{*}{$U_{2}$} & Sim. & Ana. & Sim. & Ana. & Sim. & Ana. \\
\cline { 2 - 7 } & $O P_{2}^{R A S}(1),(8)$ & $F_{G_{2}}\left(\tau_{2}\right),(10)$ & $O P_{2}^{R A S}\left(k_{1}^{*}\right),(8)$ & $F_{G_{2}}\left(\tau_{2}\right),(10)$ & $O P_{2}^{R A S}\left(k_{2}^{*}\right),(8)$ & $F_{G_{2}}^{\left(k_{2}^{*}\right)}\left(\tau_{2}\right),(33)$ \\
& $E_{x_{2}}^{R A S}(1),(18)$ & $E_{x_{2}}^{R A S}(1),(21)$ & $E_{x_{2}}^{R A S}\left(k_{1}^{*}\right),(18)$ & $E_{x_{2}}^{R A S}(1),(21)$ & $E_{x_{2}}^{R A S}\left(k_{2}^{*}\right),(18)$ & $E_{x_{2}}^{B A S-2},(34)$ \\
\hline
\end{tabular}

\section{Numerical Results}

Various numerical results are given to verify how exact the derived expressions are. We match numerical results with the analytical results in the previous sections, and provide insights for the proposed networks. We consider a scenario where the distances from the source to $U_{1}$ and $U_{2}$ are $300 \mathrm{~m}$ and $100 \mathrm{~m}$, respectively. The pathloss exponent is $\eta=3$. The density of the interference around each user is $\lambda_{1}=\lambda_{2}=10^{-5}$ interferer $/ \mathrm{m}^{2}$. The figures in this section are implemented via MATLAB 2017a. It is recommended pre-installing Statistics and Machine Learning Toolbox for the function poissrnd which helps construct Poisson point processes, in which their simulation curves are obtained by averaging at least 50,000 iterations. Within each iteration, the position of each interferer, the information channels and the interference channels are reconstructed independently. The position of the interferers affecting $U_{n}$ for each iteration are uniformly distributed inside a square area with length $10^{6} \mathrm{~m}$ around each respective user. Furthermore, the analytical curves are obtained with the help of [39] providing an exceptional algorithm for numerically evaluating the Fox H-function. Other parameters are introduced as follows:

- The number of transmit antennas, without pointing out, is set default at $K=2$.

- The target data rates of $U_{1}$ and $U_{2}$ are $\bar{R}_{1}=0.5(\mathrm{bps} / \mathrm{Hz})$ and $\bar{R}_{2}=1.5(\mathrm{bps} / \mathrm{Hz})$, respectively. Furthermore, the power allocation are $\theta_{1}=0.8$ and $\theta_{2}=0.2$.

- The shape parameters of the interference channels affecting $U_{1}$ and $U_{2}$ are $\alpha_{1}=\alpha_{2}=1$, respectively.

In Figure 2, three schemes of antenna configuration are plotted to indicate system performance in term of the outage probability versus SIR for different values of $K$. From Figure 2, at very high SIRs, a significant improvement in outage can be seen. Firstly, we see that our proposed NOMA scheme in BAS mode almost exhibit bigger gap between performance of two users due to different power allocation factors at high SIR. Secondly, outage performance in case of $K=4$ outperforms that of $K=2$. It can be observed that the number of selected transmit antenna does not change outage performance at both users. Outage in the BAS mode is better than that in the RAS mode. This result shows that tight matching between Monte-Carlo simulations and analytical simulations.

The main result is reported in Figure 3 in term of the system performance, i.e., $1-\left(1-O P_{1}^{X}\right)(1-$ $\left.O P_{2}^{X}\right)$ with $X \in\{R A S, B A S-1, B A S-2\}$. In low SIR regime, the performance of $U_{1}$ is very poor, thus our concern should focus on improving $U_{2}$. In high SIR regime, the performance of $U_{1}$ are much better, thus our target on $U_{2}$ is not good. As a result, the RAS scheme in the considered NOMA system provides the worse case or upper performance bound. It also can be perceived that in lower SIR regime, 
i.e., $25 \div 40 \mathrm{~dB}$, the proposed BAS-2 scheme for NOMA outperforms even the optimal performance of OMA, which approximates to our proposed RAS scheme.

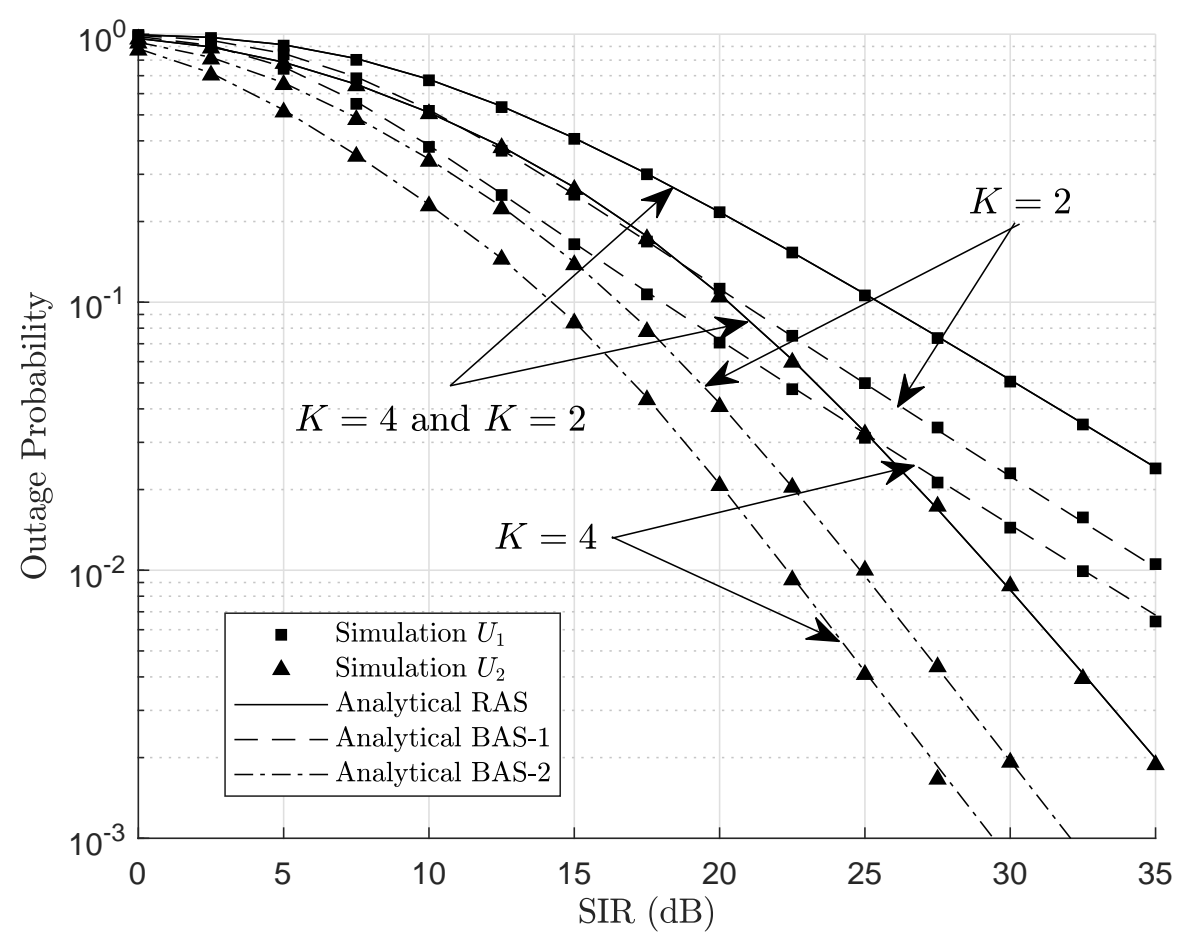

Figure 2. Outage Probability at each user versus the average SIR (dB).

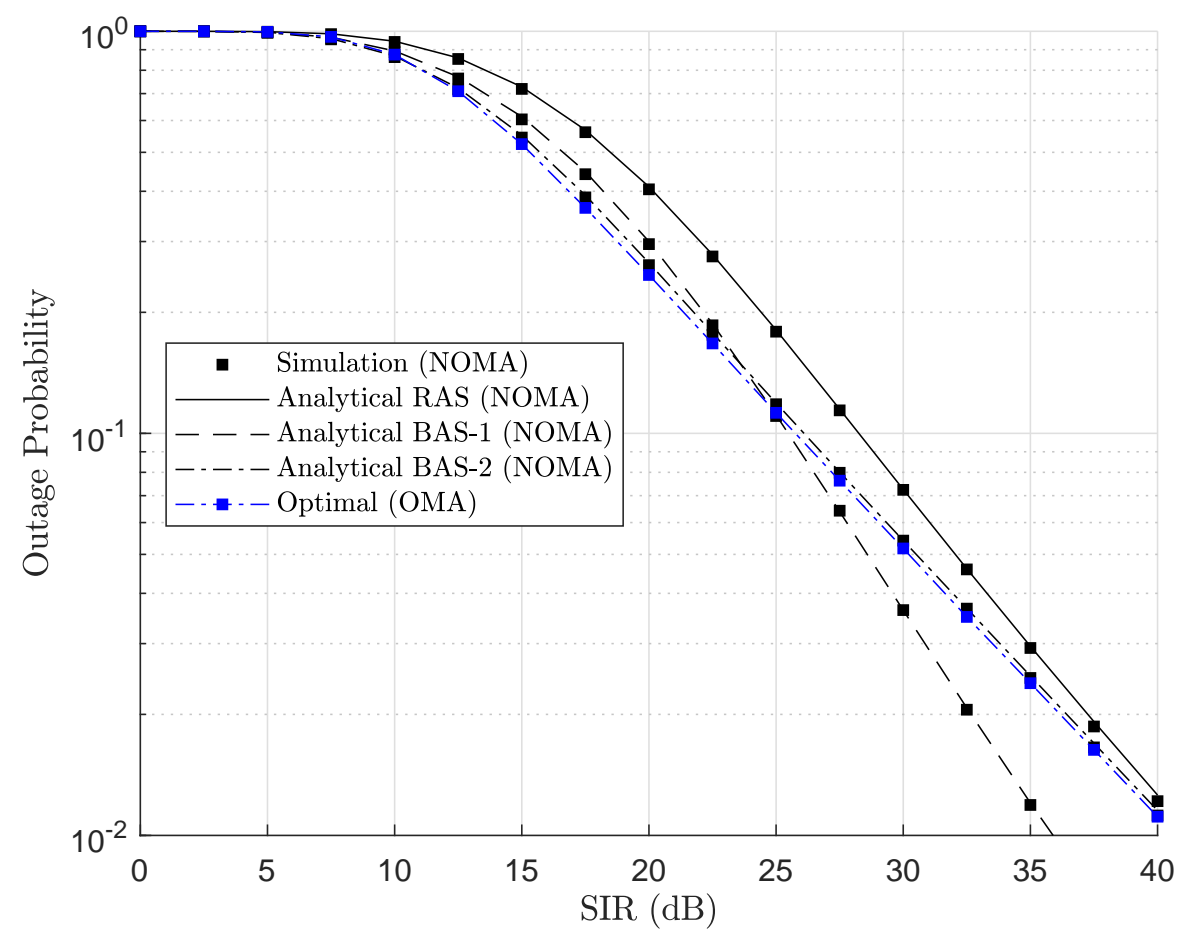

Figure 3. Outage performance of the proposed system, in which $d_{1}=200 \mathrm{~m}, d_{2}=100 \mathrm{~m}, \bar{R}_{1}=1$ (bps $/ \mathrm{Hz}$ ) and $\bar{R}_{2}=3(\mathrm{bps} / \mathrm{Hz})$. 


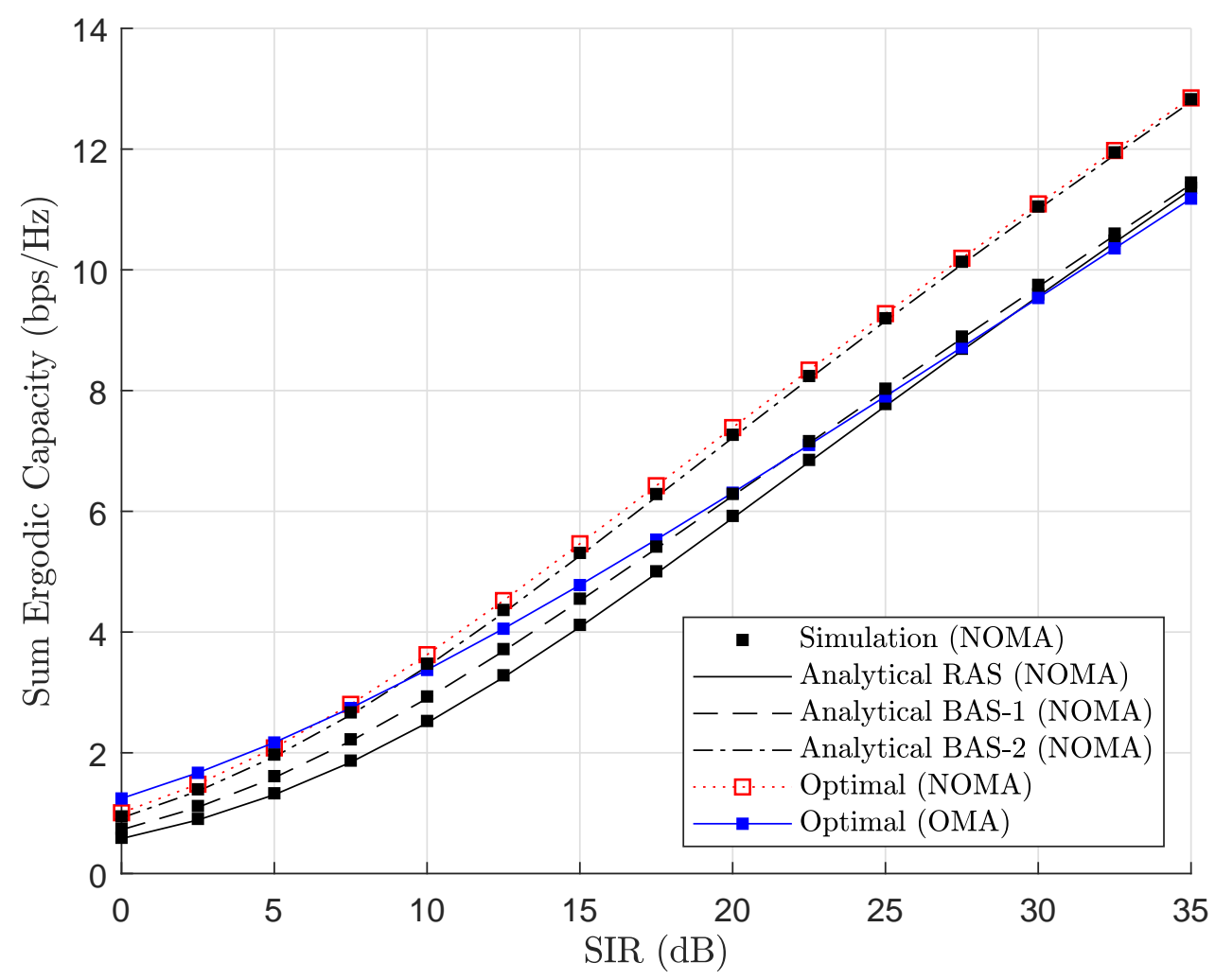

Figure 4. Sum Ergodic Capacity.

Figure 4 plots ergodic capacity as varying SIR from $0(\mathrm{~dB})$ to $35(\mathrm{~dB})$. Ergodic capacity increases significantly at high SIR, as SIR $=35(\mathrm{~dB})$. It is important to indicate that the sum Ergodic Capacity is dominant by $U_{2}$, the user with better normalized channel conditions; thus, should be focused on to optimize for obtaining optimal performance of user $U_{2}$ rather than that of user $U_{1}$. However, a slight improvement is observed at optimal ergodic capacity for user $U_{2}$. In contrast with the previous figure, lower bound of ergodic capacity happens at RAS mode. A similar trend as observed in Figure 3 is that when the average transmit SIR is relatively high, our proposed TAS schemes provide better performance than OMA.

Moreover, Figure 5 shows the ergodic capacity versus interference densities $\lambda$ and optimal ergodic capacity is indicated while Figure 6 presents the impact of interference density to outage probability. It can be clearly seen that the ergodic capacity performance degrades more steeply with an increase of $\lambda$ regardless of the increasing number of selected transmit antenna at the BS. It can be confirmed that higher ergodic capacity happens at higher number of transmit antennas, i.e., the better case as $K=8$. The ergodic capacity can be decreased to very low by increasing $\lambda$ close to -50 . Interestingly, the optimal ergodic capacity can be achieved at a slight improvement compared with the normal case. The lower bound of ergodic capacity exists at RAS scheme. This simulation result verifies the ergodic capacity declines significantly at high density of interference. Similar trend can be explained for case of outage performance as observed in Figure 6. 


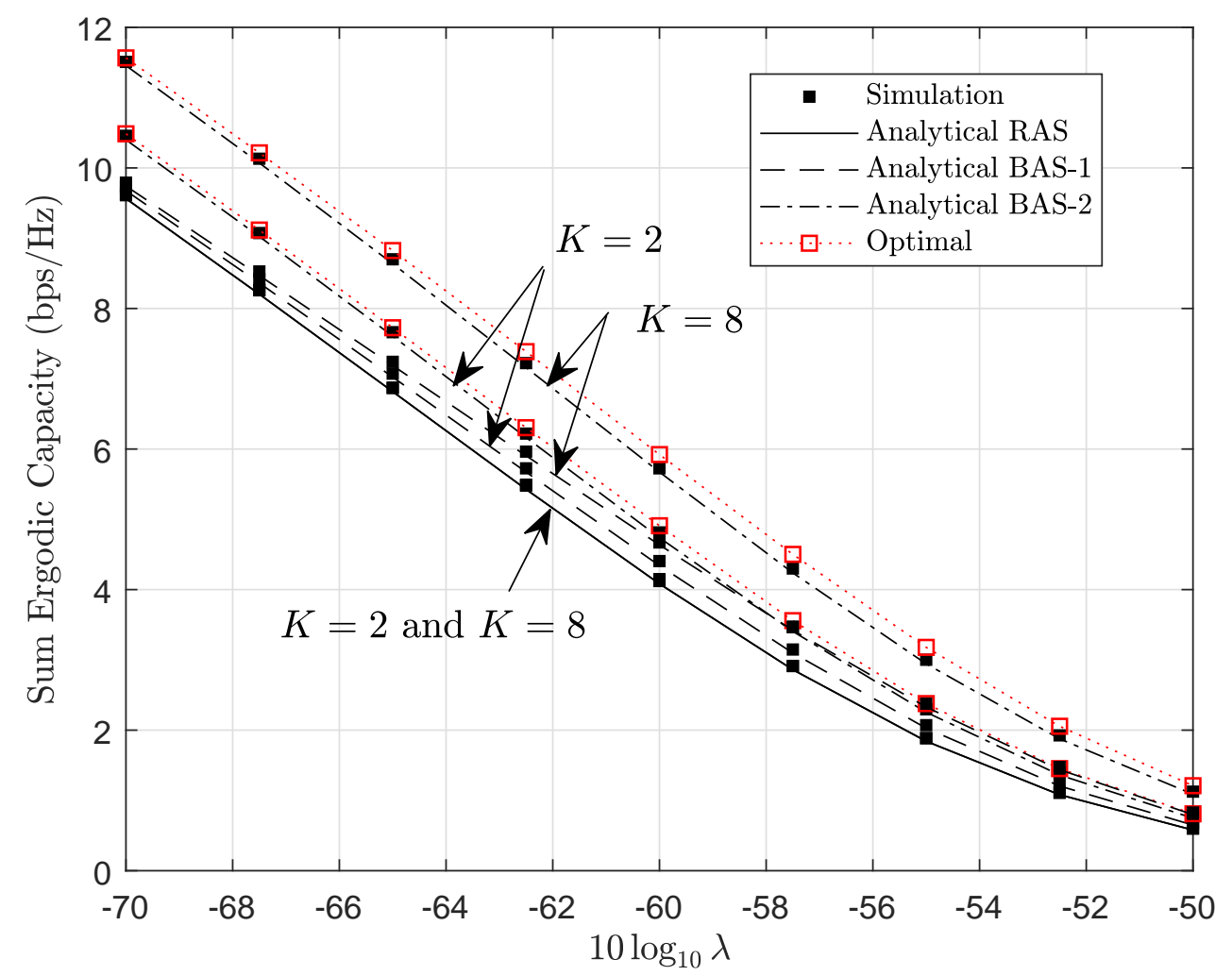

Figure 5. Sum Ergodic Capacity versus the interferer density, where $\lambda=\lambda_{1}=\lambda_{2}$ and $\bar{\gamma}=1$.

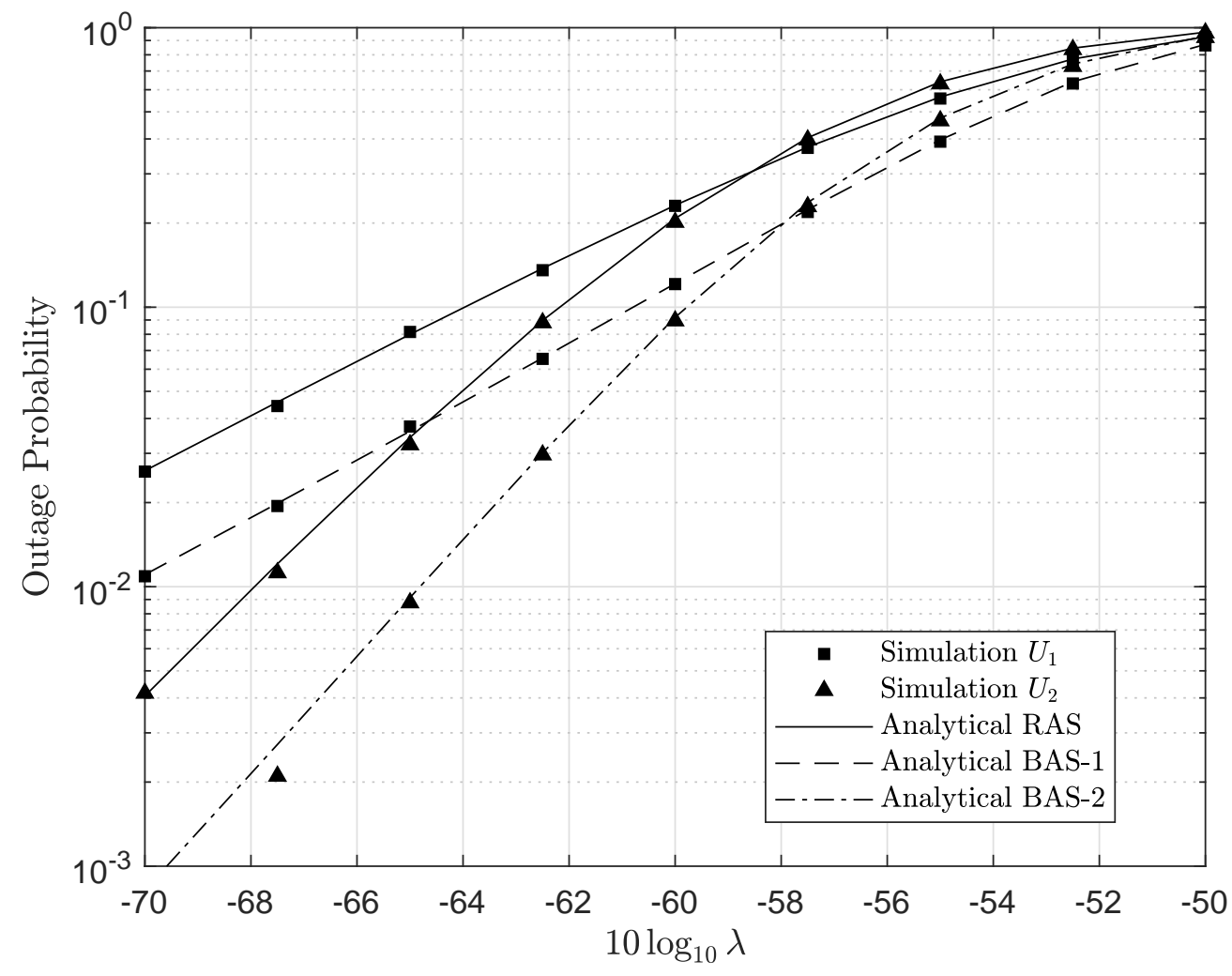

Figure 6. Outage Probability at each user versus the interferer density, where $\lambda=\lambda_{1}=\lambda_{2}$ and $\bar{\gamma}=1$. 


\section{Conclusions}

The outage performance of a NOMA system in two proposed modes for the selected antenna at the BS in the presence of a Poisson field of interferers was studied. It was shown that the interference density at near locations with each user exhibits crucial impacts on both outage probability and ergodic capacity performance. However, the end-to-end outage performance is improved by increasing the number of transmit antennas. The RAS mode is considered as a performance bound to provide characteristics of these metrics regardless of the number of antennas, while optimal performance can be achieved for the second user in such NOMA system.

Author Contributions: D.-T.D. provided idea, wrote paper and verified expressions; T.-L.N. derived mathematical problems and performed experiments; B.M.L. contributed to prepare manuscript and delivered valuable comments. All authors have read and agreed to the published version of the manuscript.

Funding: This work was supported by the National Research Foundation of Korea (NRF) grant funded by the Korea government (MSIT) (Grant No.: NRF-2019R1A4A1023746) (Grant No.: NRF-2017R1D1A1B03028350).

Conflicts of Interest: The authors declare no conflict of interest.

\section{Appendix A. Proof of Lemma 1}

First, we want to address the CDF of $X_{n}$ as

$$
F_{X_{n}}(y)=1-\exp \left(-\frac{y}{\mu_{n}}\right), \gamma>0
$$

Subsequently, the CDF of the unorderd normalized channel gain can be expressed as

$$
\begin{aligned}
F_{\bar{G}_{n}}(\gamma) & =\operatorname{Pr}\left\{X_{n}<\gamma I_{n}\right\} \\
& =\int_{0}^{\infty} F_{X_{n}}(y) f_{I_{n}}(x) d y d x \\
& =1-\int_{0}^{\infty} \exp \left(-\frac{x \gamma}{\mu_{n}}\right) f_{I_{n}}(x) d x=\mathcal{L}_{I_{n}}\left(\frac{\gamma}{\mu_{n}}\right),
\end{aligned}
$$

in which $\mathcal{L}_{I_{n}}(s)$ denotes the Laplace transform of $I_{n}$ in infinite networks. According to [...], $\mathcal{L}_{I_{n}}(s)$ under Nakgami- $m$ fading with arbitrary shape parameter $\alpha_{n}$ can be given as

$$
\mathcal{L}_{I_{n}}(s)=\exp \left(-\frac{\pi \lambda_{n}}{\Gamma\left(\alpha_{n}\right)} \Gamma\left(\alpha_{n}+\frac{2}{\eta}\right) \Gamma\left(1-\frac{2}{\eta}\right)\left(\frac{s}{\alpha_{n}}\right)^{\frac{2}{\eta}}\right), s>0 .
$$

Substituting (A5) into (A4) we then obtain the desired result, which concludes the proof.

\section{References}

1. Li, X.; Li, J.; Liu, Y.; Ding, Z.; Nallanathan, A. Residual Transceiver Hardware Impairments on Cooperative NOMA Networks. IEEE Trans. Wirel. Commun. 2019. [CrossRef]

2. $\quad \mathrm{Li}, \mathrm{X} . ; \mathrm{Li}, \mathrm{J} . ; \mathrm{Li}, \mathrm{L}$. Performance Analysis of Impaired SWIPT NOMA Relaying Networks Over Imperfect Weibull Channels. IEEE Syst. J. 2019. [CrossRef]

3. Nguyen, T.-L.; Do, D.-T. Power Allocation Schemes for Wireless Powered NOMA Systems with Imperfect CSI: System model and performance analysis. Int. J. Commun. Syst. 2018, 31, e3789. [CrossRef]

4. Li, X.; Liu, M.; Deng, C.; Mathiopoulos, P.T.; Ding, Z.; Liu, Y. Full-Duplex Cooperative NOMA Relaying Systems with I/Q Imbalance and Imperfect SIC. IEEE Wirel. Commun. Lett. 2019. [CrossRef]

5. Nguyen, T.-L.; Van Nguyen, M.S.V.; Do, D.-T.; Voznak, M. Enabling Non-Linear Energy Harvesting in Power Domain based Multiple Access in Relaying Networks: Outage and Ergodic Capacity Performance Analysis. Electronics 2019, 8, 817. [CrossRef]

6. Do, D.-T.; Van Nguyen, M.-S. Device-to-device transmission modes in NOMA network with and without Wireless Power Transfer. Comput. Commun. 2019, 139, 67-77. [CrossRef] 
7. Do, D.-T.; Le, A.-T.; Le, C.-B.; Lee, B.M. On Exact Outage and Throughput Performance of Cognitive Radio based Non-Orthogonal Multiple Access Networks with and without D2D Link. Sensors 2019, 19, 3314. [CrossRef]

8. Do, D.-T.; Le, A.-T. NOMA based cognitive relaying: Transceiver hardware impairments, relay selection policies and outage performance comparison. Comput. Commun. 2019, 146, 144-154. [CrossRef]

9. Do, D.-T.; Le, A.-T.; Lee, B.-M. On Performance Analysis of Underlay Cognitive Radio-Aware Hybrid OMA/NOMA Networks with Imperfect CSI. Electronics 2019, 8, 819. [CrossRef]

10. Do, D.-T.; Nguyen, M.-S.V.; Hoang, T.-A.; Lee, B.M. Exploiting Joint Base Station equipped Multiple Antenna and Full-Duplex D2D users in Power Domain Division Based Multiple Access Networks. Sensors 2019, 19, 2475. [CrossRef]

11. Do, D.-T.; Le, C.-B.; Lee, B.M. Robust Transmit Antenna Design for Performance Improvement of Cell-Edge User: Approach of NOMA and Outage/Ergodic Capacity Analysis. Sensors 2019, 19, 4907. [CrossRef] [PubMed]

12. Le, C.-B.; Do, D.-T. Joint Evaluation of Imperfect SIC and Fixed Power Allocation Scheme for Wireless Powered D2D-NOMA networks with multiple antennas at Base Station. Wirel. Netw. 2019, 25, 5069-5081. [CrossRef]

13. Al-Hussaibi, W.A.; Ali, F.H. Efficient User Clustering, Receive Antenna Selection, and Power Allocation Algorithms for Massive MIMO-NOMA Systems. IEEE Access 2019, 7, 31865-31882. [CrossRef]

14. Khan, A.S.; Chatzigeorgiou, I.; Lambotharan, S.; Zheng, G. Network-Coded NOMA with Antenna Selection for the Support of Two Heterogeneous Groups of Users. IEEE Trans. Wirel. Commun. 2019, 18, 1332-1345. [CrossRef]

15. Lv, L.; Ni, Q.; Ding, Z.; Chen, J. Application of non-orthogonal multiple access in cooperative spectrumsharing networks over Nakagami-m fading channels. IEEE Trans. Veh. Technol. 2017, 66, 5506-5511. [CrossRef]

16. Ma, Z.; Zhang, Z.; Ding, Z.; Fan, P.; Li, H. Key techniques for 5G wireless communications: Network architecture, physical layer, and MAC layer perspectives. Sci. China Inf. Sci. 2015, 58, 1-20. [CrossRef]

17. Yang, Z.; Ding, Z.; Fan, P.; Ma, Z. Outage performance for dynamic power allocation in hybrid non-orthogonal multiple access systems. IEEE Commun. Lett. 2016, 20, 1695-1698. [CrossRef]

18. Li, X.; Li, C.; Jin, Y. Dynamic resource allocation for transmit power minimization in OFDM-based NOMA systems. IEEE Commun. Lett. 2016, 20, 2558-2561. [CrossRef]

19. Yang, Z.; Ding, Z.; Fan, P.; Al-Dhahir, N. A general power allocation scheme to guarantee quality of service in downlink and uplink NOMA systems. IEEE Trans. Wirel. Commun. 2016, 15, 7244-7257. [CrossRef]

20. Zhang, X.; Gao, Q.; Gong, C.; Xu, Z. User grouping and power allocation for NOMA visible light communication multi-cell networks. IEEE Commun. Lett. 2017, 21, 777-780. [CrossRef]

21. Zhu, X.; Jiang, C.; Kuang, L.; Ge, N.; Lu, J. Non-orthogonal multiple access based integrated terrestrialsatellite networks. IEEE J. Sel. Areas Commun. 2017, 35, 2253-2267. [CrossRef]

22. Yan, X.; Xiao, H.; Wang, C.-X.; An, K. Outage performance of NOMAbased hybrid satellite-terrestrial relay networks. IEEE Wireless Commun. Lett. 2018, 7, 538-541. [CrossRef]

23. Yan, X.; Xiao, H.; An, K.; Zhen, G.; Tao, W. Hybrid satellite terrestrial relay networks with cooperative non-orthogonal multiple access. IEEE Commun. Lett. 2018, 22, 978-981 [CrossRef]

24. Chen, Z.; Yuan, J.; Vucetic, B. Analysis of transmit antenna selection/maximal-ratio combining in Rayleigh fading channels. IEEE Trans. Veh. Technol. 2005, 54, 1312-1321. [CrossRef]

25. Zhang, Y.; Ge, J.; Serpedin, E. Performance analysis of nonorthogonal multiple access for downlink networks with antenna selection over Nakagami-m fading channels. IEEE Trans. Veh. Technol. 2017, 66, 10590-10594. [CrossRef]

26. Yu, Y.; Chen, H.; Li, Y.; Ding, Z.; Song, L.; Vucetic, B. Antenna selection for MIMO nonorthogonal multiple access systems. IEEE Trans. Veh. Technol. 2018, 67, 3158-3171. [CrossRef]

27. Liu, X.; Wang, X. Efficient antenna selection and user scheduling in 5G massive MIMO-NOMA system. In Proceedings of the 2016 IEEE 83rd Vehicular Technology Conference (VTC Spring), Nanjing, China, 15-18 May 2016; pp. 1-5.

28. Yu, Y.; Chen, H.; Li, Y.; Ding, Z.; Zhuo, L. Antenna selection in MIMO cognitive radio-inspired NOMA systems. IEEE Commun. Lett. 2017, 21, 2658-2661. [CrossRef] 
29. Liu, Y.; Qin, Z.; Elkashlan, M.; Gao, Y.; Hanzo, L. Enhancing the physical layer security of non-orthogonal multiple access in large-scale networks. IEEE Trans. Wirel. Commun. 2017, 16, 1656-1672. [CrossRef]

30. Ding, Z.; Fan, P.; Poor, H.V. Random beamforming in millimeter-wave NOMA networks. IEEE Access 2017, 5, 7667-7681. [CrossRef]

31. Zhou, Y.; Wong, V.W.S.; Schober, R. Dynamic decode-and-forward based cooperative NOMA with spatially random users. IEEE Trans. Wirel. Commun. 2018, 17, 3340-3356. [CrossRef]

32. Zhou, Y.; Wong, V.W.S.; Schober, R. Stable Throughput Regions of Opportunistic NOMA and Cooperative NOMA With Full-Duplex Relaying. IEEE Trans. Wirel. Commun. 2018, 17, 5059-5075. [CrossRef]

33. Heath, R.W.; Kountouris, M.; Bai, T. Modeling heterogeneous network interference using Poisson point processes. IEEE Trans. Signal Process. 2013, 61, 4114-4126. [CrossRef]

34. Cardieri, P. Modeling interference in wireless ad hoc networks. IEEE Commun. Surv. Tutor. 2010, 12, 551-572. [CrossRef]

35. Haenggi, M.; Ganti, R.K. Interference in large wireless networks. Found. Trends Netw. 2009, 3, 127-248. [CrossRef]

36. Lai, X.; Zou, W.; Xie, D.; Li, X.; Fan, L. DF relaying networks with randomly distributed interferers. IEEE Access 2017, 5, 18909-18917. [CrossRef]

37. Ganti, R.K.; Haenggi, M. Spatial and temporal correlation of the interference in ALOHA ad hoc networks. IEEE Commun. Lett. 2009, 13, 631-633. [CrossRef]

38. Haenggi, M. Diversity loss due to interference correlation. IEEE Commun. Lett. 2012, 16, 1600-1603. [CrossRef]

39. Soulimani, A.; Benjillali, M.; Chergui, H.; da Costa, D.B. On Multihop Weibull-Fading Communications Performance Analysis Framework and Applications. arXiv 2016, arXiv:1610.08535.

(C) 2019 by the authors. Licensee MDPI, Basel, Switzerland. This article is an open access article distributed under the terms and conditions of the Creative Commons Attribution (CC BY) license (http:/ / creativecommons.org/licenses/by/4.0/). 